\title{
PM functions, their characteristic intervals and iterative roots
}

\author{
by WeINIAN Zhang (Chengdu)
}

\begin{abstract}
The concept of characteristic interval for piecewise monotone functions is introduced and used in the study of their iterative roots on a closed interval.

I. Introduction. The iterative root of order $n$ of a function $F: E \rightarrow E$, for a given positive integer $n$ and a given set $E$, is a function $f: E \rightarrow E$ such that

$$
f^{n}=F,
$$

where $f^{n}$ denotes the $n$th iterate of $f$, i.e., $f^{n}=f \circ f^{n-1}$ and $f^{0}=$ id.

The problem of iterative roots, as an important subject in the theory of functional equations, has been studied deeply in various aspects, for example, for real functions by Bödewadt [2], Fort [4] and Kuczma [7-9], and for complex functions by Kneser [5] and Rice [10], since Babbage [3], Abel [1] and Koenigs [6] initiated that research in the last century. In particular, the research in this field gets very active in Poland and China.

It is well known that a strictly increasing continuous function has continuous iterative roots of any order but a strictly decreasing function has no continuous iterative roots of even order. In particular, for monotone functions we have the following result.

Theorem (Bödewadt [2]). Let $F: I=[a, b] \rightarrow I$ be continuous and strictly increasing. Then for any integer $n \geq 2$ and $A, B \in(a, b)$ with $A<B$, (1.1) has a continuous and strictly increasing solution $f$ on $I$ satisfying $F(a) \leq f(A)<f(B) \leq F(b)$.
\end{abstract}

However, there are few results without monotonicity assumptions.

1991 Mathematics Subject Classification: 39B12, 39B22.

Key words and phrases: iterative root, piecewise monotone function, characteristic interval. 
In 1993, while visiting Poland, the author had a talk about an interesting method, presented in Chinese by J. Zhang and L. Yang [11], based on introducing the so-called "characteristic interval" for piecewise monotone functions. In this paper this method is presented in detail. In Section II we discuss the properties of this type of functions; Section III is devoted to the notion of characteristic interval and an extension theorem; finally, in Section IV, the results of Section III are applied to give the existence of iterative roots for piecewise monotone functions (abbreviated as PM functions) on $I=[a, b] \subset \mathbb{R}^{1}$. In Sections II to IV, all considered functions are supposed to be continuous from $I$ into itself.

\section{PM functions}

Definition 1. An interior point $x_{0}$ in $I$ is referred to as a monotone point of $F: I \rightarrow I$ if $F$ is strictly monotone in a neighborhood of $x_{0}$. Otherwise, $x_{0}$ is called a fort (or a non-monotone point). Furthermore, $F \in C^{0}(I, I)$ is referred to as a strictly piecewise monotone function or $P M$ function if $F$ has only finitely many forts in $I$. Let $N(F)$ denote the number of forts of $F$, and $\operatorname{PM}(I, I)$ the set of all continuous PM functions from $I$ into itself.

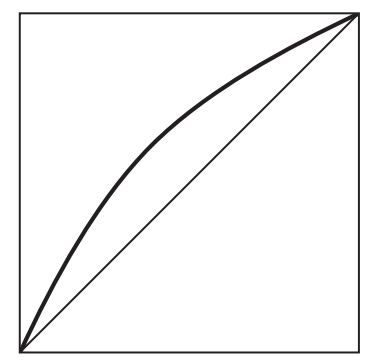

Fig. 1. No fort

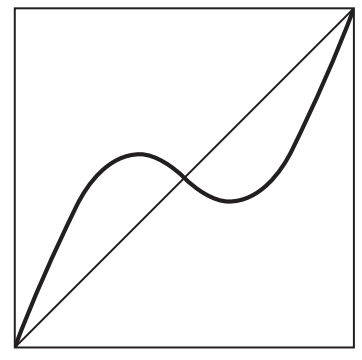

Fig. 2. A PM function

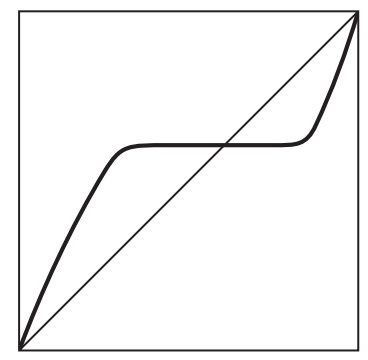

Fig. 3. Infinitely many forts

From Figure 3 we see that a fort may not be an extreme point.

LEMma 2.1 (equivalent definition). An interior point $x_{0}$ in $I$ is a fort of $F$ iff for any $\varepsilon>0$ there are two points $x_{1}, x_{2}$ in I with $x_{1} \neq x_{2},\left|x_{1}-x_{0}\right|<\varepsilon$ and $\left|x_{2}-x_{0}\right|<\varepsilon$ such that $F\left(x_{1}\right)=F\left(x_{2}\right)$.

The simple proof is omitted.

Lemma 2.2. (i) If $F_{1}, F_{2} \in \mathrm{PM}(I, I)$ then $F_{2} \circ F_{1} \in \mathrm{PM}(I, I)$.

(ii) If $F_{2} \circ F_{1} \in \operatorname{PM}(I, I)$ then $F_{1} \in \operatorname{PM}(I, I)$. Here $\circ$ denotes the composition of functions. 
Proof. Let $S, S_{1}$, and $S_{2}$ denote the sets of forts of $F=F_{2} \circ F_{1}, F_{1}$, and $F_{2}$ respectively, and let $S_{3}=\left\{x \in I \mid F_{1}(x) \in S_{2}\right\}$. Clearly,

$$
S=S_{1} \cup S_{3} .
$$

It follows that the cardinal numbers satisfy

$$
\begin{aligned}
\# S & \leq \# S_{1}+\# S_{3}, \\
\# S_{1} & \leq \# S .
\end{aligned}
$$

Thus (2.3) implies (ii)

On the other hand, $\# S_{1}<\infty$ and $\# S_{2}<\infty$ imply $\# S_{3}<\infty$; otherwise, by $\# S_{2}<\infty$, there are infinitely many $x_{1}<x_{2}<\ldots<x_{n}<\ldots$ in $I$ such that $F_{1}\left(x_{i}\right)=F_{1}\left(x_{j}\right), i \neq j$. By Lemma 2.1 this contradicts the fact that $\# S_{1}<\infty$ and implies (i) by (2.2).

Corollary 2.3. If $f^{n} \in \operatorname{PM}(I, I)$ then $f \in \operatorname{PM}(I, I)$; and vice versa.

Furthermore, (2.3) implies for $F \in \operatorname{PM}(I, I)$ that

$$
0=N\left(F^{0}\right) \leq N(F) \leq N\left(F^{2}\right) \leq N\left(F^{3}\right) \leq \ldots \leq N\left(F^{n}\right) \leq \ldots
$$

Let $H(F)$ denote the smallest positive integer $k$ such that $N\left(F^{k}\right)=N\left(F^{k+1}\right)$, and let $H(F)=\infty$ when (2.4) is a strictly increasing sequence.

Lemma 2.4. Let $F_{1}, F_{2} \in \operatorname{PM}(I, I)$. Then $N\left(F_{2} \circ F_{1}\right)=N\left(F_{1}\right)$ iff $F_{2}$ is strictly monotone on $[m, M]$, the range of $F_{1}$, where $m=\min F_{1}$ and $M=\max F_{1}$.

Proof. We use the notations $S, S_{1}, S_{2}, S_{3}, F$, etc. as in the proof of Lemma 2.2. Note that $[m, M]$ is not a single point set since $F_{1}$ as a PM function is not constant. On the one hand, suppose $F_{2}$ is strictly monotone on $[m, M]$. For each $x_{0} \in S_{3}$, by the monotonicity of $F_{2}, F_{1}\left(x_{0}\right)=m$ or $M$, that is, $x_{0}$ is an extreme point and, of course, a fort of $F_{1}$. Thus $S_{3} \subset S_{1}$. From (2.1), $S=S_{1}$ and $N\left(F_{2} \circ F_{1}\right)=N\left(F_{1}\right)$.

On the other hand, for an indirect proof of the necessity we assume that $F_{2}$ has a fort $x_{1}$ in $[m, M]$. The continuity of $F_{1}$ implies that there is a monotone point $x_{0} \in(a, b)$ such that $F_{1}\left(x_{0}\right)=x_{1}$, i.e., $x_{0} \in S_{3} \backslash S_{1}$. Thus $S \backslash S_{1} \neq \emptyset$, i.e., $N\left(F_{2} \circ F_{1}\right) \neq N\left(F_{1}\right)$. This gives a contradiction.

Lemma 2.5. Let $F \in \operatorname{PM}(I, I)$ and $H(F)=k<\infty$. Then for any integer $i>0, N\left(F^{k}\right)=N\left(F^{k+i}\right)$.

Proof. Let $m_{i}$ and $M_{i}$ denote the minimum and maximum of $F^{i}$ on $I$ respectively. Since $H(F)=k$ implies

$$
N\left(F^{k}\right)=N\left(F^{k+1}\right)=N\left(F \circ F^{k}\right),
$$

by Lemma $2.4, F$ is strictly monotone on $\left[m_{k}, M_{k}\right]$. However,

$$
m_{k} \leq m_{k+i-1}<M_{k+i-1} \leq M_{k} \quad \text { for } i \geq 1,
$$


so $F$ is also strictly monotone on $\left[m_{k+i-1}, M_{k+i-1}\right]$. By Lemma 2.4,

$$
N\left(F^{k+i-1}\right)=N\left(F^{k+i}\right), \quad i=1,2, \ldots
$$

This completes the proof.

Lemma 2.6. If $H(F)=k$, then $H\left(F^{i}\right)=[k / i]+\operatorname{sgn}\{k / i\}$, for every integer $i>0$, where $[x]$ denotes the largest integer not exceeding $x$ and $\{x\}=x-[x]$.

Proof. Let $F_{1}=F^{i}$ and let $r$ denote the right side of the above expression for $H\left(F^{i}\right)$. Since

$$
i(r-1)<k \leq i r<i(r+1),
$$

we get

$$
N\left(F_{1}^{r-1}\right)=N\left(F^{i(r-1)}\right)<N\left(F^{k}\right)=N\left(F_{1}^{r}\right)=N\left(F_{1}^{r+1}\right) .
$$

This implies $H\left(F_{1}\right)=r$ and completes the proof.

Theorem 1. Let $F \in \operatorname{PM}(I, I)$ and $H(F)>1$. Then $F$ has no continuous iterative roots of order $n$ for $n>N(F)$.

Proof. Assume $f \in C^{0}(I, I)$ is an iterative root of order $n$. By Corollary 2.3, $f \in \operatorname{PM}(I, I)$. Since $H(F)>1$, i.e., $N\left(f^{2 n}\right)=N\left(F^{2}\right)>N(F)=$ $N\left(f^{n}\right)$, we see that $H(f)>n$ and $0=N\left(f^{0}\right)<N(f)<N\left(f^{2}\right)<\ldots<$ $N\left(f^{n}\right)$. This implies $N\left(f^{n}\right) \geq n$, i.e., $N(F) \geq n$, contrary to the assumption.

Problem 1. It is still an open question whether $F$ has an iterative root of order $n$ for all $n \leq N(F)$.

This theorem says that $F$ can have continuous iterative roots of infinitely many orders only when $H(F) \leq 1$. In what follows, we concentrate on the case where $H(F) \leq 1$. We show that on the so-called characteristic interval this case reduces to the monotone case.

III. Characteristic interval. Suppose $H(F) \leq 1$. For $F$ non-monotone it follows that $N(F)=N\left(F^{2}\right)$. By Lemma $2.4, F$ is strictly monotone on $[m, M]$, where $m=\min F$ and $M=\max F$. Obviously, extending appropriately the interval on which $F$ is monotone, one can find two points $a^{\prime}, b^{\prime} \in I$, $a^{\prime}<b^{\prime}$, such that

(i) $a^{\prime}$ and $b^{\prime}$ are either forts or endpoints;

(ii) there is no fort inside $\left(a^{\prime}, b^{\prime}\right)$;

(iii) $\left[a^{\prime}, b^{\prime}\right] \supset[m, M]$.

Definition 2. The unique interval $\left[a^{\prime}, b^{\prime}\right]$ obtained above is referred to as the characteristic interval of $F$. 


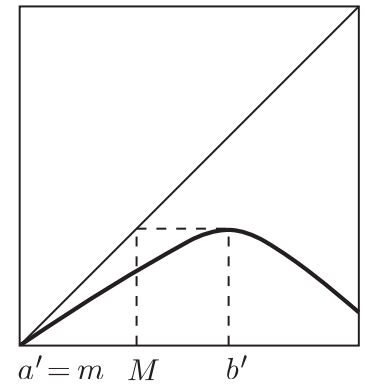

Fig. 4

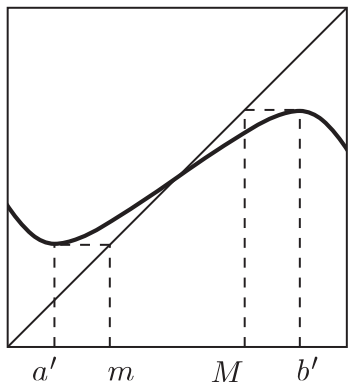

Fig. 6

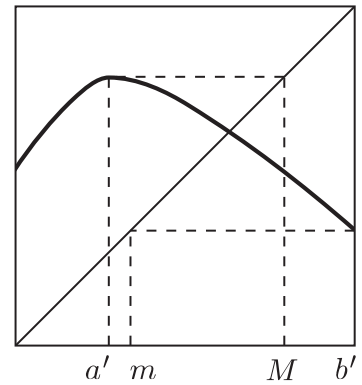

Fig. 5

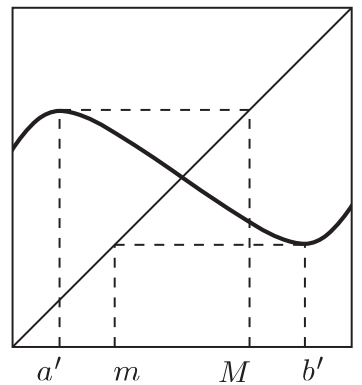

Fig. 7

The above figures illustrate the cases where $H(F) \leq 1$.

Theorem 2. Let $F \in \operatorname{PM}(I, I)$ and $H(F) \leq 1$. Suppose $F$ has a continuous iterative root $f$ of order $n>1$. Then

(i) $F$ is strictly monotone from $\left[a^{\prime}, b^{\prime}\right]$ into itself;

(ii) all periodic points of $F$ are inside $\left[a^{\prime}, b^{\prime}\right]$;

(iii) all periodic points of $f$ are inside $\left[a^{\prime}, b^{\prime}\right]$;

(iv) $f$ is strictly monotone from $\left[a^{\prime}, b^{\prime}\right]$ into itself;

(v) $f^{n}(x)=F(x)$ for $x \in\left[a^{\prime}, b^{\prime}\right]$;

(vi) if $n>N(F)+1$ and $F\left(x^{\prime}\right)=a^{\prime}$ or $b^{\prime}$ for some $x^{\prime} \in I$, then $x^{\prime} \in$ $\left[a^{\prime}, b^{\prime}\right]$.

Proof. We use the same notations $m_{i}$ and $M_{i}$ as in the proof of Lemma 2.5. Obviously, the sequence $\left\{m_{i}\right\}$ is non-decreasing and $\left\{M_{i}\right\}$ is non-increasing. Then (i) follows from the definition of characteristic interval, in particular from $\left[m_{1}, M_{1}\right] \subset\left[a^{\prime}, b^{\prime}\right]$. By Corollary $2.3, f$ is also strictly monotone on $\left[a^{\prime}, b^{\prime}\right]$. To prove (ii), let $x_{0}$ be a periodic point of $F$. Then for some integer $k>0, x_{0}=F^{k}\left(x_{0}\right) \in\left[m_{k}, M_{k}\right] \subset\left[m_{1}, M_{1}\right] \subset\left[a^{\prime}, b^{\prime}\right]$. Now (iii) follows from (ii) since all periodic points of $f$ are periodic points of $F$.

Concerning (iv), it suffices to prove that $f(x) \in\left[a^{\prime}, b^{\prime}\right]$ for $x \in\left[a^{\prime}, b^{\prime}\right]$. In case $f$ is increasing on $\left[a^{\prime}, b^{\prime}\right]$, for an indirect proof we assume, without 
loss of generality, that $f\left(a^{\prime}\right)<a^{\prime}$. Since $f(a) \geq a$, the continuity implies $f\left(x_{1}\right)=x_{1}$ for some $x_{1} \in\left[a, a^{\prime}\right)$, i.e., $f$ has a periodic point outside $\left[a^{\prime}, b^{\prime}\right]$. This contradicts (iii).

On the other hand, in case $f$ is decreasing on $\left[a^{\prime}, b^{\prime}\right], F$ is strictly monotone on $\left[f\left(b^{\prime}\right), f\left(a^{\prime}\right)\right]$; otherwise, $F$ has forts in this interval, and by Lemma 2.2, $f^{n+1}(x)=F(f(x))$ and even $F(F(x))=f^{2 n}(x)$ have forts on $\left[a^{\prime}, b^{\prime}\right]$, which implies $N\left(F^{2}\right)>N(F)$, contrary to $H(F) \leq 1$. Furthermore, neither $a^{\prime}$ nor $b^{\prime}$ is an interior point of $\left[f\left(b^{\prime}\right), f\left(a^{\prime}\right)\right]$ since $a^{\prime}$ and $b^{\prime}$ are forts (or endpoints) of $F$. Thus, in order to prove $\left[f\left(b^{\prime}\right), f\left(a^{\prime}\right)\right] \subset\left[a^{\prime}, b^{\prime}\right]$ we show that the interior of $\left[a^{\prime}, b^{\prime}\right] \cap\left[f\left(b^{\prime}\right), f\left(a^{\prime}\right)\right]$ is not empty. Indeed, otherwise $F(f(x))=f(F(x))$ cannot reach the interior of $\left[a^{\prime}, b^{\prime}\right]$ for all $x \in I$. However, $\left(a^{\prime}, b^{\prime}\right) \supset\left(m_{1}, M_{1}\right) \neq \emptyset$, so $F(f(x))$ must reach the interior of $\left[a^{\prime}, b^{\prime}\right]$ for some $x \in I$. This contradiction completes the proof of (iv). (iv) yields (v) naturally.

Finally, we prove (vi). Note that $n>N(F)+1>N(F)$ implies $H(f)<$ $n$; otherwise, $N\left(f^{n}\right)>N\left(f^{n-1}\right)>\ldots>N(f)>N\left(f^{0}\right)=0$, which yields a contradiction that $N\left(f^{n}\right) \geq n>N(F)$. It follows that $N\left(f^{n-1}\right)=N(F)$ and by Lemma 2.5 that $N\left(f^{n-1}\right)=N\left(f^{n-1} \circ f^{n-1}\right)$, i.e., $H\left(f^{n-1}\right) \leq 1$. Thus $\left[a^{\prime}, b^{\prime}\right]$ is also the characteristic interval of $f^{n-1}$ and $f^{n-1}$ maps $I$ into it, since $\left[\min f^{n-1}, \max f^{n-1}\right] \supset\left[m_{1}, M_{1}\right]$ and by Lemma 2.2 (or from (2.1)), $f^{n-1}$ and $f^{n}(=F)$ have common forts. Therefore, the fact that $F=f \circ f^{n-1}$ reaches $a^{\prime}$ (or $\left.b^{\prime}\right)$ on $I$ implies that $f$ also reaches $a^{\prime}$ (or $\left.b^{\prime}\right)$ on $\left[a^{\prime}, b^{\prime}\right]$. In particular, when $f$ is increasing on $\left[a^{\prime}, b^{\prime}\right]$ we can assert that $f\left(a^{\prime}\right)=a^{\prime}$ (or $\left.f\left(b^{\prime}\right)=b^{\prime}\right)$, and then $F\left(a^{\prime}\right)=a^{\prime} \quad\left(\right.$ or $\left.F\left(b^{\prime}\right)=b^{\prime}\right)$.

Now, we consider the case where $f$ is decreasing on $\left[a^{\prime}, b^{\prime}\right]$. Using the same arguments as above, by the hypothesis that $n>N(F)+1$ we have $H(f)<n-1$ and $H\left(f^{n-2}\right) \leq 1$, i.e., $f^{n-2}$ maps $I$ into $\left[a^{\prime}, b^{\prime}\right]$. Thus the fact that $F=f^{2} \circ f^{n-1}$ reaches $a^{\prime}$ (or $b^{\prime}$ ) on $I$ implies that $f^{2}$ also reaches $a^{\prime}$ (or $b^{\prime}$ ) on $\left[a^{\prime}, b^{\prime}\right]$. Since $f$ is decreasing on $\left[a^{\prime}, b^{\prime}\right]$ and $f\left(a^{\prime}\right) \leq b^{\prime}, f\left(b^{\prime}\right) \geq a^{\prime}$, we see that $f\left(a^{\prime}\right)=b^{\prime}$ and $f\left(b^{\prime}\right)=a^{\prime}$, i.e., $f$ maps $\left[a^{\prime}, b^{\prime}\right]$ onto itself. This implies that $F$ also maps $\left[a^{\prime}, b^{\prime}\right]$ onto itself. Of course, $F$ reaches $a^{\prime}$ (or $b^{\prime}$ ) on $\left[a^{\prime}, b^{\prime}\right]$. This completes the proof.

Theorem 3 (extension). Suppose $F \in \operatorname{PM}(I, I)$ and $H(F) \leq 1$. Let $\left[a^{\prime}, b^{\prime}\right]$ be the characteristic interval, let $m$ and $M$ denote the minimum and maximum of $F$ on $[a, b]$, and $m^{\prime}$ and $M^{\prime}$ those on $\left[a^{\prime}, b^{\prime}\right]$. If restricted to $\left[a^{\prime}, b^{\prime}\right]$, equation (1.1) has a continuous solution $f_{1}$ which maps $\left[a^{\prime}, b^{\prime}\right]$ into itself and maps $[m, M]$ into $\left[\mathrm{m}^{\prime}, M^{\prime}\right]$, then there exists a continuous function $f$ from $I$ into $I$ such that

(i) $f(x)=f_{1}(x)$ for all $x \in\left[a^{\prime}, b^{\prime}\right]$, and

(ii) $f$ satisfies (1.1) on the whole interval $I$. 
This theorem says that the problem of iterative roots can be reduced to that for monotone functions on a subinterval.

Pro of. Let $F_{1}$ be the restriction of $F$ to $\left[a^{\prime}, b^{\prime}\right]$. By Theorem 2, its inverse $F_{1}^{-1}:\left[m^{\prime}, M^{\prime}\right] \rightarrow\left[a^{\prime}, b^{\prime}\right]$ is continuous. Let

$$
f=F_{1}^{-1} \circ f_{1} \circ F
$$

on $I$. Because $F(x) \in[m, M] \subset\left[a^{\prime}, b^{\prime}\right]$ for $x \in I$ and $f_{1}(y) \in\left[m^{\prime}, M^{\prime}\right]$ for $y \in[m, M]$, the definition in (3.1) is reasonable and $f:[a, b] \rightarrow\left[a^{\prime}, b^{\prime}\right]$ is continuous. Obviously, for $x \in I$,

$$
f^{n}(x)=\left(F_{1}^{-1} \circ f_{1}^{n} \circ F\right)(x)=\left(F_{1}^{-1} \circ F_{1} \circ F\right)(x)=F(x) .
$$

This completes the proof.

\section{Existence of iterative roots}

Theorem 4. Let $F \in \operatorname{PM}(I, I)$ and $H(F) \leq 1$. Suppose

(a) $F$ is increasing on its characteristic interval $\left[a^{\prime}, b^{\prime}\right]$, and

(b) $F(x)$ on $I$ cannot reach $a^{\prime}$ and $b^{\prime}$ unless $F\left(a^{\prime}\right)=a^{\prime}$ or $F\left(b^{\prime}\right)=b^{\prime}$.

Then for any integer $n>1, F$ has a continuous iterative root of order $n$. Moreover, these conditions are necessary for $n>N(F)+1$.

Proof. By Bödewadt's theorem stated in Section I, $F$ on $\left[a^{\prime}, b^{\prime}\right]$ has a continuous iterative root $f_{1}$ of order $n$, which satisfies $m^{\prime}=F\left(a^{\prime}\right) \leq f_{1}(m)<$ $f_{1}(M) \leq F\left(b^{\prime}\right)=M^{\prime}$. By Theorem 3, equation (1.1) has a continuous solution $f$ on the whole interval $I$, which is an extension of $f_{1}$. In particular, for $n>N(F)+1$, by Theorem 2(vi), the condition (b) is necessary. Furthermore, it is well known that a strictly decreasing function has no continuous iterative roots of even order, so (a) is also necessary.

Problem 2. Does $F$ have iterative roots of order $n$ for $n \leq N(F)+1$ when $H(F) \leq 1$ and $F\left(x^{\prime}\right)=a^{\prime}$ (or $b^{\prime}$ ) for some $x^{\prime} \in I$ but $x^{\prime} \notin\left[a^{\prime}, b^{\prime}\right]$ ?

Theorem 5. Suppose $F \in \operatorname{PM}(I, I), H(F) \leq 1$, and $F$ is decreasing on its characteristic interval $\left[a^{\prime}, b^{\prime}\right]$. If either $F\left(a^{\prime}\right)=b^{\prime}$ and $F\left(b^{\prime}\right)=a^{\prime}$, or $a^{\prime}<F(x)<b^{\prime}$ on $I$, then for any odd $n>0, F$ has an iterative root of order $n$, and for even $n,(1.1)$ has no continuous solutions.

Proposition. Suppose $F:\left[a^{\prime}, b^{\prime}\right] \rightarrow\left[a^{\prime}, b^{\prime}\right]$ is continuous and decreasing, and either $F\left(a^{\prime}\right)=b^{\prime}$ and $F\left(b^{\prime}\right)=a^{\prime}$, or $a^{\prime}<F(x)<b^{\prime}$ on $\left[a^{\prime}, b^{\prime}\right]$. Then for $n \geq 1$,

$$
f^{2 n+1}=F
$$


has a decreasing $C^{0}$ solution $f$ on $\left[a^{\prime}, b^{\prime}\right]$ such that

$$
F\left(b^{\prime}\right) \leq f(M)<f(m) \leq F\left(a^{\prime}\right),
$$

where $m=\min F$ and $M=\max F$.

Proof. Since (4.2) is trivial when $F\left(a^{\prime}\right)=b^{\prime}$ and $F\left(b^{\prime}\right)=a^{\prime}$, we only prove the proposition under the condition that $a^{\prime}<F(x)<b^{\prime}$ on $\left[a^{\prime}, b^{\prime}\right]$. Note that $F$ has a unique fixed point $x_{0}$ in $\left(a^{\prime}, b^{\prime}\right)$ and

$$
a^{\prime} \leq m<x_{0}<M \leq b^{\prime} .
$$

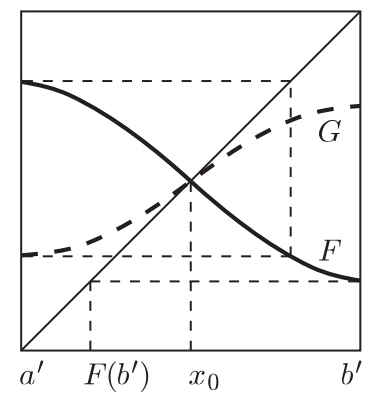

Fig. 8

Clearly, $G=F^{2}$ is $C^{0}$ and increasing on $\left[a^{\prime}, b^{\prime}\right]$ and certainly on $\left[a^{\prime}, x_{0}\right]$. By Bödewadt's theorem (see Section I), the equation

$$
g^{2}=G
$$

has an increasing $C^{0}$ solution $g$ on $\left[a^{\prime}, x_{0}\right]$ such that

$$
G\left(a^{\prime}\right)=g\left(F\left(b^{\prime}\right)\right) \text {. }
$$

Here (4.5) is guaranteed by the fact that $a^{\prime}<F\left(b^{\prime}\right)<x_{0}$. Furthermore, the monotonicity implies that $G(x)>x$ and then $g(x)>x$ on $\left[a^{\prime}, x_{0}\right]$, and that $G\left(x_{0}\right)=x_{0}$ and $g\left(x_{0}\right)=x_{0}$, so $g$ maps the subinterval $\left[F\left(b^{\prime}\right), x_{0}\right]$ into itself. By Bödewadt's theorem, the equation

$$
h^{2 n+1}=g
$$

also has an increasing $C^{0}$ solution $h$ on $\left[F\left(b^{\prime}\right), x_{0}\right]$ such that

$$
g\left(F\left(b^{\prime}\right)\right) \leq h(\beta)
$$

for $\beta:=\min \{g(m), F(M)\}$. Here (4.7) is guaranteed by the fact that $\beta>$ $\min \left\{g\left(a^{\prime}\right), F\left(b^{\prime}\right)\right\}=F\left(b^{\prime}\right)$, since (4.5) associated with (4.4) implies that

$$
g\left(a^{\prime}\right)=F\left(b^{\prime}\right) .
$$

In particular, because $g$ is continuous and increasing on $\left[a^{\prime}, x_{0}\right]$ and $\left[a^{\prime}, x_{0}\right] \supset$ $\left[F\left(b^{\prime}\right), x_{0}\right]$, using Bödewadt's inductive construction of iterative roots, one 
can extend the solution $h$ of (4.6) to the whole interval $\left[a^{\prime}, x_{0}\right]$, i.e., $h$ is increasing and continuous on $\left[a^{\prime}, x_{0}\right]$ and (4.6) still holds. Let

$$
h_{1}(x)= \begin{cases}h(x), & x \in\left[a^{\prime}, x_{0}\right], \\ F^{-1} \circ h \circ F(x), & x \in\left(x_{0}, b^{\prime}\right] .\end{cases}
$$

Clearly, $h_{1}$ is increasing and $C^{0}$ on $\left[a^{\prime}, b^{\prime}\right]$ and

$$
h_{1}^{2 n+1}=g, \quad h_{1} \circ F=F \circ h_{1} .
$$

Let

$$
f(x)=h_{1}^{-2 n} \circ F(x), \quad x \in\left[a^{\prime}, b^{\prime}\right] .
$$

Obviously, $f$ is decreasing and $C^{0}$ on $\left[a^{\prime}, b^{\prime}\right]$, and

$$
\begin{aligned}
f^{2 n+1} & =h_{1}^{-2 n(2 n+1)} \circ F^{2 n+1} \quad(\text { by }(4.10)) \\
& =g^{-2 n} \circ F^{2 n} \circ F=F,
\end{aligned}
$$

that is, $f$ is a solution of $(4.1)$ on $\left[a^{\prime}, b^{\prime}\right]$.

Moreover, on $\left[x_{0}, b^{\prime}\right]$,

$$
f(x)=h^{-2 n} \circ F ;
$$

on $\left[a^{\prime}, x_{0}\right]$, the range of $F$ is contained by $\left[x_{0}, b^{\prime}\right]$ and then

$$
\begin{aligned}
f(x) & =h_{1}^{-2 n} \circ F=\left(F^{-1} \circ h \circ F\right)^{-2 n} \circ F \\
& =\left(F^{-1} \circ h^{-1} \circ F\right)^{2 n} \circ F=F^{-1} \circ h^{-2 n} \circ F^{2} \\
& =F^{-1} \circ h^{-2 n} \circ G=F^{-1} \circ h^{2 n+2} .
\end{aligned}
$$

Thus the inequalities in (4.2), that is,

$$
f(m) \leq F\left(a^{\prime}\right), \quad f(M) \geq F\left(b^{\prime}\right),
$$

are equivalent to

$$
h^{2 n+2}(m) \geq G\left(a^{\prime}\right), \quad h^{-2 n} \circ F(M) \geq F\left(b^{\prime}\right),
$$

and to

$$
\begin{aligned}
h(g(m)) & \geq G\left(a^{\prime}\right) \\
h(F(M)) & \geq g\left(F\left(b^{\prime}\right)\right)=G\left(a^{\prime}\right) \quad(\text { by }(4.5)) .
\end{aligned}
$$

Obviously (4.16) and (4.17) hold by (4.7) and by the monotonicity of $h$. This completes the proof.

Proof of Theorem 5. For odd $n$, the result follows easily from the above Proposition and Theorem 3. For even $n$, the result is obvious since a strictly decreasing function has no iterative roots of even order, as stated in Section I.

By Theorem 3 (extension theorem), many known results for monotone functions can be generalized to PM functions under the hypothesis that $H(F) \leq 1$. 
Acknowledgements. The author thanks the referee for his helpful comments.

\section{References}

[1] N. H. A bel, Oeuvres Complètes, t. II, Christiania, 1881, 36-39.

[2] U. T. Bödewadt, Zur Iteration reeller Funktionen, Math. Z. 49 (1944), 497-516.

[3] J. M. Dubbey, The Mathematical Work of Charles Babbage, Cambridge Univ. Press, 1978.

[4] M. K. Fort Jr., The embedding of homeomorphisms in flows, Proc. Amer. Math. Soc. 6 (1955), 960-967.

[5] H. Kneser, Reelle analytische Lösungen der Gleichung $\varphi(\varphi(x))=e^{x}$ und verwandter Funktionalgleichungen, J. Reine Angew. Math. 187 (1950), 56-67.

[6] G. Koenigs, Recherches sur les intégrales de certaines équations fonctionnelles, Ann. Ecole Norm. Sup. (3) 1 (1884), Suppl., 3-41.

[7] M. Kuczma, Functional Equations in a Single Variable, Monografie Mat. 46, PWN, Warszawa, 1968.

[8] —, Fractional iteration of differentiable functions, Ann. Polon. Math. 22 (1969/70), $217-227$.

[9] M. Kuczma and A. Smajdor, Fractional iteration in the class of convex functions, Bull. Acad. Polon. Sci. Sér. Sci. Math. Astronom. Phys. 16 (1968), 717-720.

[10] R. E. Rice, B. Schweizer and A. Sklar, When is $f(f(z))=a z^{2}+b z+c$ ?, Amer. Math. Monthly 87 (1980), 252-263.

[11] J. Zhang and L. Yang, Discussion on iterative roots of piecewise monotone functions, Acta Math. Sinica 26 (1983), 398-412 (in Chinese).

Centre for Math. Sciences

CICA, Academia Sinica

Chengdu 610041, P.R. China

Reçu par la Rédaction le 14.11.1994

Révisé le 11.9.1995 